\title{
Upregulation of IGF1 by tumor-associated macrophages promotes the proliferation and migration of epithelial ovarian cancer cells
}

\author{
LI LIU, XINJING WANG, XIAODUAN LI, XIAOLI WU, MEILING TANG and XIPENG WANG \\ Department of Gynecology, Shanghai First Maternity and Infant Hospital, \\ Tongji University School of Medicine, Shanghai 201204, P.R. China
}

Received June 2, 2017; Accepted December 4, 2017

DOI: $10.3892 /$ or.2017.6148

\begin{abstract}
Ovarian cancer (OC), of which epithelial ovarian cancer (EOC) is the most common, is the deadliest gynecological tumor because of the difficulties in detection at early stages, and metastasis and chemoresistance at advanced stages. Tumor-associated macrophages (TAMs) differentiate through alternative pathways and play important roles in tumor growth and metastasis. However, the underlying mechanism remains unclear. Here, we established a mouse TAM model using bone marrow monocytes and conditioned medium (CM) of TAMs to culture ID8 mouse EOC cells. The results showed that TAM CM accelerated the proliferation and migration of ID8 cells. In a previous study, gene chip analysis showed that human TAMs expressed significantly higher levels of insulinlike growth factor-1 (IGF1) than undifferentiated M0 myeloid cells. In the present study, we observed that the IGF1 level was higher in human EOC specimens than that in benign ovarian tumor specimens, and further analysis showed that a higher level of IGF1 was related to more advanced clinical stage and liver metastasis. Therefore, we hypothesized that TAMs may accelerate the proliferation and migration of EOC cells by upregulating IGF1. As expected, increased IGF1 expression at both the mRNA and protein levels was observed in ID8 cells cultured with TAM CM, whereas blockade of the IGF1 pathway in ID8 cells with an IGF1 neutralizing antibody effectively reversed the promotion of proliferation and migration. Finally, we inhibited the phosphorylation of insulin-like
\end{abstract}

Correspondence to: Professor Xipeng Wang, Department of Gynecology, Shanghai First Maternity and Infant Hospital, Tongji University School of Medicine, 2699 Gaoke Road, Pudong, Shanghai 201204, P.R. China

E-mail: wangxipeng@xinhuamed.com.cn

Abbreviations: OC, ovarian cancer; EOC, epithelial ovarian cancer; IGF1, insulin-like growth factor-1; IGF1R, insulin-like growth factor-1 receptor; TAMs, tumor-associated macrophages; $\mathrm{CM}$, conditioned medium; TME, tumor microenvironment; CAFs, cancer-associated fibroblasts

Key words: tumor-associated macrophages, epithelial ovarian cancer, IGF1, proliferation, migration growth factor-1 receptor (IGF1R) and its downstream molecules Akt and Erk with the IGF1R inhibitor linsitinib, and observed that the treatment effectively suppressed the proliferation and migration of ID8 cells exposed to TAM CM. Thus, we demonstrated that TAMs may promote the growth and metastasis of EOC via the activation of the IGF1 pathway; thus, targeting the IGF1 pathway may be promising for EOC therapy.

\section{Introduction}

Ovarian cancer (OC) is the most fatal gynecological malignant tumor, causing 151,900 deaths worldwide in 2012 (1). According to recent statistics, OC is still the fifth leading cause of cancer-related female deaths in the United States (2). Because there are no effective methods to screen OC, $75 \%$ of cases are diagnosed at an advanced stage, with tumor cells spreading widely throughout the abdominal cavity (3). Although many therapeutic methods, including surgery and cisplatin-based chemotherapy, have been used, severe metastasis and chemoresistance lead to a 5-year overall survival rate in no more than $25-35 \%$ of cases $(3,4)$. EOC accounts for $90 \%$ of OC (5); therefore, it is important to investigate how this cancer progresses.

Although cancer cells unlimitedly proliferate and survive, a tumor microenvironment (TME) is required for the formation and growth of clinically relevant tumors (6). The TME, comprising mesenchymal stem cells, cancer-associated fibroblasts (CAFs), myeloid cells, mesothelial cells and factors released by these cells, contributes to tumor growth, immune escape, distant metastasis and chemoresistance of cancer (6-8). For example, CAF-derived exosomes (tiny vesicles formed during endocytosis and $30-150 \mathrm{~nm}$ in size) can promote the survival and proliferation of pancreatic cancer cells, thus affecting responses to the standard chemotherapeutic agent gemcitabine (9). Mesothelial cells stimulated with TGF- $\beta$ can promote $\mathrm{OC}$ cell attachment and proliferation by activating the promoters of matrix metalloprotein-2 and matrix metalloprotein-9 (10).

TAMs, the most common immune cells in the TME (11), primarily refer to macrophages infiltrating into tumor tissues (12). These cells are derived from circulating monocytes and induced to differentiate through alternative pathways through various factors in the TME, mainly due 
to the activation of the Notch pathway $(11,12)$. In addition to the microenvironment within tumor tissues, TAMs are also distributed in some special organs and lymph nodes, associated with metastasis to these regions $(13,14)$. TAMs infiltrate into OC tissues in large numbers (15-17), contributing to the progression of OC $(18,19)$, thus negatively affecting the progression-free survival rates and overall survival rates of these patients (16). However, the precise mechanism of how TAMs contribute to the progression of OC remains unclear.

The IGF1 pathway comprises three receptor tyrosine kinases [IGF-1R, insulin-like growth factor-2 receptor (IGF-2R), and insulin receptor (IR)], three ligands (insulin, IGF-1, and IGF-2), and six serum insulin-like growth factor binding proteins (IGFBPs), which are important regulators of this pathway (20). This pathway can enhance the proliferation and development of cells by initiating the anti-apoptotic PI3K/Akt/mTOR and the mitogenic Ras/Raf/Mek/Erk pathways $(21,22)$. The IGF1 pathway is associated with the growth, metastasis and clinical outcome of various cancers, including prostate cancer, gastric cancer, lung cancer and breast cancer (23-27). For example, CAFs can increase the invasion ability of pancreatic cancer cells via paracrine IGF1/ IGF1R signaling, particularly under hypoxia (27), and IGF1R is highly expressed in chronic lymphocytic leukemia cells, whereas the inhibition of IGF1R can enhance the death of CLL cells (28).

The IGF1 pathway has also been associated with the progression of OC. Women carrying haplotype $2 \mathrm{C}$ of the IGF1 gene have a decreased risk of $\mathrm{OC}$, whereas those carrying haplotype 1D or 2D have an increased risk (29). Serous ovarian carcinoma cells are strongly positive for IGF1, and IGF1 could downregulate the expression of E-cadherin and upregulate Snail and Slug expression, thereby promoting the epithelial to mesenchymal transition of human OC cells $(30,31)$. Low IGFBP-3 expression is clinically correlated with high tumor grade, advanced stage and poor survival of ovarian endometrioid cancer patients (32). The IGF1/PI3K/NFkB/Erk pathway was found to be upregulated in ovarian specimens of patients demonstrating relative resistance compared with those demonstrating sensitivity (33). Furthermore, high circulating IGF-1 levels have been correlated with decreased OC risk, and overall and progression-free survival were significantly prolonged in patients with higher serum IGF1 levels (34-36). However, the underlying reason for the abnormality of IGF1 in OC patients remains unknown.

In the present study, we showed that TAMs enhanced the proliferation and migration of mouse OC ID8 cells by upregulating IGF1, and inhibition of the IGF1 pathway using an IGF1 inhibitor effectively suppressed the proliferation and migration of ID8 cells exposed to TAM-conditioned medium (CM). These results indicate that targeting the IGF1 pathway is a promising EOC therapy.

\section{Materials and methods}

TAM model establishment. A TAM model was established according to Lin et al (37), and the protocols for the treatment of animals were approved by the Ethics Committee of Tongji University prior to the study. The C57 mice were euthanized, and their hind legs were removed and placed in $75 \%$ alcohol for $5 \mathrm{~min}$. Soft tissues were removed, a $26-\mathrm{G}$ needle attached to a 1-cc syringe was inserted into the bone marrow cavity to wash out cells with RPMI-1640 (Gibco, Foster city, CA, USA) containing $10 \%$ fetal bovine serum (FBS; Gibco) until the bone became white. The medium with bone marrow cells was passed through a cell strainer with $70-\mu$ m pores (Merck Millipore, Billerica, MA, USA) into a 50-ml centrifuge tube. The cells were centrifuged for $10 \mathrm{~min}$ at 1,350 rpm, and the supernatant was subsequently discarded. Next, the cells were resuspended in $5 \mathrm{ml}$ RPMI-1640 supplemented with $10 \%$ FBS and centrifuged for $10 \mathrm{~min}$ at $1,350 \mathrm{rpm}$. The cells were resuspended in RPMI-1640 containing 10\% FBS and $1 \%$ penicillin-streptomycin (Gibco) to a final concentration of $5 \times 10^{6}$ cells $/ \mathrm{ml}$. Then, $1 \times 10^{7}$ cells/well were plated in 6 -well plates. M-CSF (10 ng/ml; R\&D Systems, Minneapolis, MN, USA) was added to the medium for M0 cell formation, or M-CSF, IL-4, IL-13, and IL-10 (10 ng/ml; R\&D Systems) were added to the medium for TAM formation. The cells were incubated at $37^{\circ} \mathrm{C}$ in a humidified, $5 \% \mathrm{CO}_{2}$ incubator, and medium containing cytokines was changed daily for 4 days.

Cell line. ID8 mouse EOC cells were purchased from Fuheng Biotechnology Co., Ltd. (Shanghai, China) and maintained in DMEM (Gibco) medium supplemented with 10\% FBS and $1 \%$ penicillin-streptomycin. The cells were cultured at $37^{\circ} \mathrm{C}$ in a humidified, $5 \% \mathrm{CO}_{2}$ incubator.

TAM CM preparation, transfer and coculture. The TAM CM transfer was performed according to Richards et al (9). TAM medium was centrifuged at 2,500 rpm for $30 \mathrm{~min}$ to remove cell debris and then mixed with complete DMEM at the ratio of 1:1. Complete RPMI-1640, and DMEM was mixed at the ratio of 1:1 as normal medium. TAM CM or normal medium was transferred to plates with ID8 cells for coculture daily. IGF1 neutralizing antibody (monoclonal, rabbit to mouse; Abcam, Cambridge, MA, USA) was dissolved in PBS and linsitinib (Selleck, Houston, TX, USA) was dissolved in DMSO before use.

RNA collection and quantitative real-time PCR ( $q R T-P C R)$. Total RNA was extracted from macrophages and ID8 cells using TRIzol reagent (Invitrogen, Carlsbad, CA, USA) according to the manufacturer's instructions. Reverse transcription was performed using a Prime Script ${ }^{\mathrm{TM}}$ II 1st Strand cDNA Synthesis kit (Takara Biotechnology Co., Ltd., Dalian, China) according to the manufacturer's instructions. qRT-PCR was performed using Talent qPCR PreMix (SYBR-Green) (Tiangen Biotech Co., Ltd., Beijing, China) and the StepOnePlus ${ }^{\mathrm{TM}}$ Real-time PCR system (Invitrogen) according to the manufacturer's instructions. The following primer sequences were used: CD204 forward, 5'-TGGAGGAGAGAATCGAAAGCA-3' and reverse, 5'-CTGGACTGACGAAATCAAGGAA-3'; IGF1 forward, 5'-CACATCATGTCGTCTTCACACC-3' and reverse, 5'-GGAAGCAACACTCATCCACAATG-3'; and GAPDH forward, 5'-AGGTCGGTGTGAACGGATTTG-3' and reverse, 5'-GGGTCGTTGATGGCAACA-3'. The final concentration of all reagents was $2 \mathrm{X}$ Talent qPCR PreMix (with SYBR-Green I), 1X; 50X ROX Reference Dye, 5X; and forward and reverse primers: $0.3 \mu \mathrm{M}$. The PCR reactions cycling conditions included an initial cycle at $95^{\circ} \mathrm{C}$ for $15 \mathrm{sec}$, followed by 40 cycles of $95^{\circ} \mathrm{C}$ for $5 \mathrm{sec}$ and $60^{\circ} \mathrm{C}$ for $15 \mathrm{sec}$. 
The data were calculated using the $2^{-\Delta \Delta \mathrm{Cq}}$ method, with GAPDH as an internal normalization control.

Western blot analyses. Total protein was collected from M0 cells, TAMs, ID8 cells treated with or without TAM CM or linsitinib using RIPA lysis buffer (Beyotime, Shanghai, China). A BCA kit (Thermo Fisher, Rockford, IL, USA) was used for protein quantification according to the manufacturer's instructions. Protein was separated on $10 \%$ SDS-PAGE gels (EpiZyme, Shanghai, China) at a consistent voltage of $80 \mathrm{~V}$ in the stacking gel and $120 \mathrm{~V}$ in the separating gel and subsequently transferred onto polyvinylidene difluoride membranes at consistent current of $300 \mathrm{~mA}$ for $80 \mathrm{~min}$. The membranes were blocked in TBST buffer with $5 \%$ bovine serum albumin (BSA) for $2 \mathrm{~h}$ at room temperature. Then, the membranes were incubated with the following primary antibodies at $4^{\circ} \mathrm{C}$ overnight: IGF1 (cat. no. ab9572; 1:1,000, monoclonal, rabbit to mouse; Abcam), CD204 (cat. no. ab15707; 1:1,000, polyclonal, rabbit to mouse; Abcam), IGF1R (cat. no. 9750; 1:1,000, monoclonal, rabbit to mouse; Cell Signaling Technology, Inc., Danvers, MA, USA), phosphoIGF1R (cat. no. 3918; 1:1,000, monoclonal, rabbit to mouse; Cell Signaling Technology, Inc.), Akt (cat. no. 4685; 1:1,000, monoclonal, rabbit to mouse; Cell Signaling Technology, Inc.), phospho-Akt (cat. no. ab81283; 1:1,000, monoclonal, rabbit to mouse; Abcam), Erk (cat. no. 4695; 1:1,000, monoclonal, rabbit to mouse; Cell Signaling Technology, Inc.), phosphoErk (cat. no. 4370; 1:1,000, monoclonal, rabbit to mouse; Cell Signaling Technology, Inc.), GAPDH (cat. no. AB0036; 1:5,000; AB2000, monoclonal, rabbit to mouse; Abways, Shanghai, China), and the HRP-conjugated rabbit secondary antibody (cat. no. 16402-1-AP; 1:5,000; Proteintech, Wuhan, China) was used to incubate the membranes at room temperature for $1 \mathrm{~h}$. The protein signals were detected using enhanced chemiluminescent HRP substrate (Merck Millipore).

Immunohistochemistry (IHC). Ovarian benign tumor specimens (patient ages ranged from 21 to 70 years) and EOC specimens (patient ages ranged from 33 to 74 years) used for tissue microarrays were obtained from the specimen repository of Shanghai First Maternity and Infant Hospital, after obtaining consent from each patient and the Ethics Committee of Tongji University. IHC was performed using an IGF1 primary antibody (1:125, monoclonal, rabbit to mouse; Abcam). Specimens (4- $\mu \mathrm{m}$ thick) were subjected to heat-induced epitope retrieval and then dewaxed in xylene and hydrated through a graded series of alcohol. The specimens were incubated in $0.3 \% \mathrm{H}_{2} \mathrm{O}_{2}$ for $30 \mathrm{~min}$ to inactivate the endogenous peroxidase. Next, the specimens were incubated with $1 \%$ goat serum (Invitrogen) for $20 \mathrm{~min}$ at room temperature, followed by incubation with IGF1 antibody overnight at $4^{\circ} \mathrm{C}$ and then rabbit secondary antibody for $1 \mathrm{~h}$ at $37^{\circ} \mathrm{C}$. A Vectastain $\mathrm{ABC}$ Elite kit (Vector Laboratories, Burlingame, CA, USA) was used according to the manufacturer's instructions for color development. The specimens were counterstained with hematoxylin and then dehydrated through a graded series of alcohol, cleared in xylene and mounted. Tumor cells with immunohistochemical expression in the cytoplasm were regarded as IGF1-positive, and the expression level was determined using the IRS system by multiplication of the staining intensity $(0$, no; 1 , weak;
2, moderate; and 3, strong staining) and the percentage of positively stained cells ( 0 , no staining; $1,<10 \%$ of cells; $2,11-50 \%$ of cells; $3,51-80 \%$ of cells; and $4,>81 \%$ of cells stained), according to Remmele and Stegner (38). The total score per sample therefore ranged from 0 to 12 ; a score $<6$ indicates low expression, whereas a score of 6-12 indicates high expression. The slides were examined in a blinded manner by two experienced investigators.

MTS proliferation assay. ID8 cells $\left(1.5 \times 10^{3}\right)$ were seeded onto 96-well plates and cultured with $100 \mu \mathrm{l}$ of medium for $72 \mathrm{~h}$. Then, $20 \mu 1$ of MTS Solution Reagent (PR Omega Biosciences, USA) was added to each well followed by incubation at $37^{\circ} \mathrm{C}$ in $5 \% \mathrm{CO}_{2}$ for $2 \mathrm{~h}$. The absorbance was measured at $490 \mathrm{~nm}$ using a 96-well plate reader. Each group had six duplicates.

Cell migration assays. Transwell chambers (Corning, Glendale, AZ, USA) containing $8-\mu \mathrm{m}$ inserts were used to measure the migration of tumor cells. ID8 cells $\left(5 \times 10^{4}\right.$ cells) in $200 \mu \mathrm{l}$ DMEM containing 2\% FBS was plated in the top chambers. The bottom of the wells was filled with $800 \mu$ l complete DMEM containing 10\% FBS. Calcein AM (Invitrogen) was used to stain the cells in the bottom of the filter membrane. The images of cells were captured using a fluorescence microscope at x100. ImageJ software was used to count the cells that had migrated to the bottom of the filter membrane. Each group had two duplicates, and five images of different fields for each duplicate were captured.

Statistical analysis. All experiments were independently performed three or more times. Statistical analysis was performed using SPSS 19.0 (SPSS, Inc., Chicago, IL, USA). The data are expressed as the means \pm SEMs or means \pm SDs and analyzed using Student's t-test or the Mann-Whitney test. The correlation of IGF1 expression and clinical and pathological factors was analyzed using the Chi-square test or the Fisher's exact test. P-value $<0.05$ was considered statistically significant.

\section{Results}

The TAM model is established using mouse bone marrow monocytes. TAMs have been characterized as a popularized M2 macrophage phenotype different from the M1 type, which differentiates through a classic pathway. In the present study, a TAM model was established using isolated primary mouse bone marrow cells. Monocytes were induced to become undifferentiated M0 cells using MCSF. To induce M0 cells to differentiate into TAMs, IL-10, IL-13, and IL-4 were continuously used.

To determine whether the TAM model was successful, analyses of cell morphology and the expression level of the TAM-specific marker CD204 was used. As shown, TAMs were larger and more stacked in morphology (Fig. 1A). The expression of CD204 was higher in TAMs at both the mRNA (Fig. 1B) and protein levels (Fig. 1C).

TAM CM enhances the proliferation and migration of ID8 cells. As TAMs are associated with the enhanced progression of various cancers, including OC, and the number of TAMs in 
A
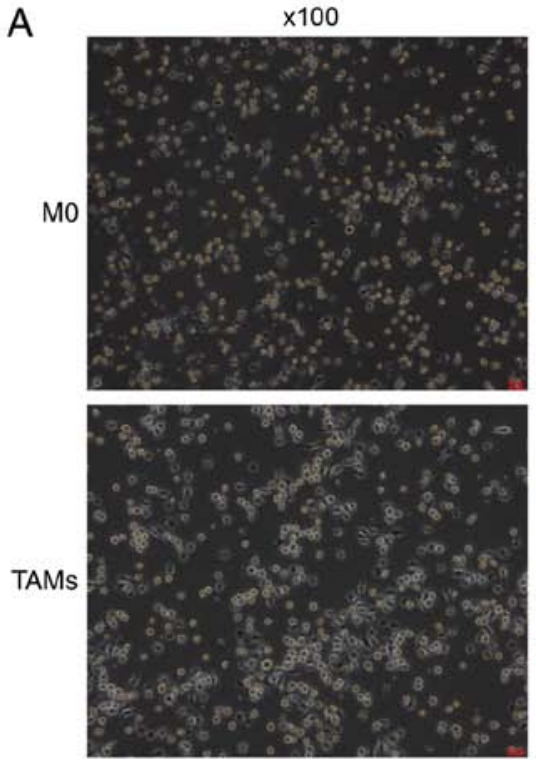
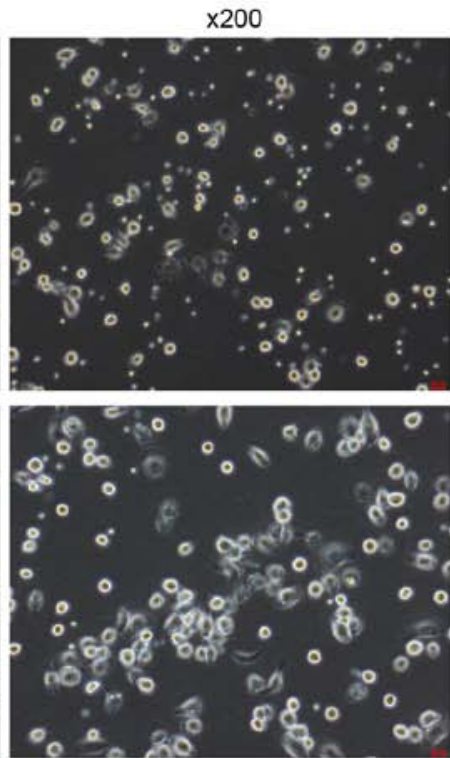

B

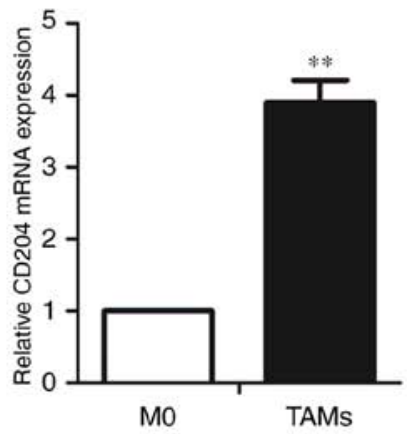

C

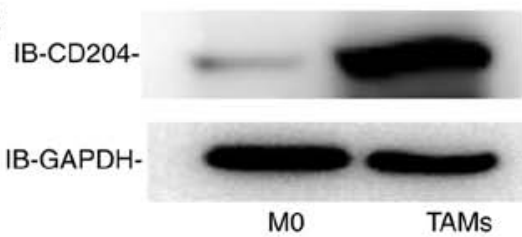

Figure 1. Mouse bone marrow monocytes are induced to become TAMs. (A) Morphology parameters, including shape, length and size, were compared between M0 cells and TAMs using a microscope. (B and C) Expression level of the TAM-specific marker CD204 was compared between M0 cells and TAMs using qRT-PCR and western blotting. The bar graphs represent the means \pm SDs of three independent experiments; ${ }^{* *} \mathrm{P}<0.001$. TAMs, tumor-associated macrophages. qRT-PCR, quantitative real-time PCR. Scale bar, $10 \mu \mathrm{m}$.
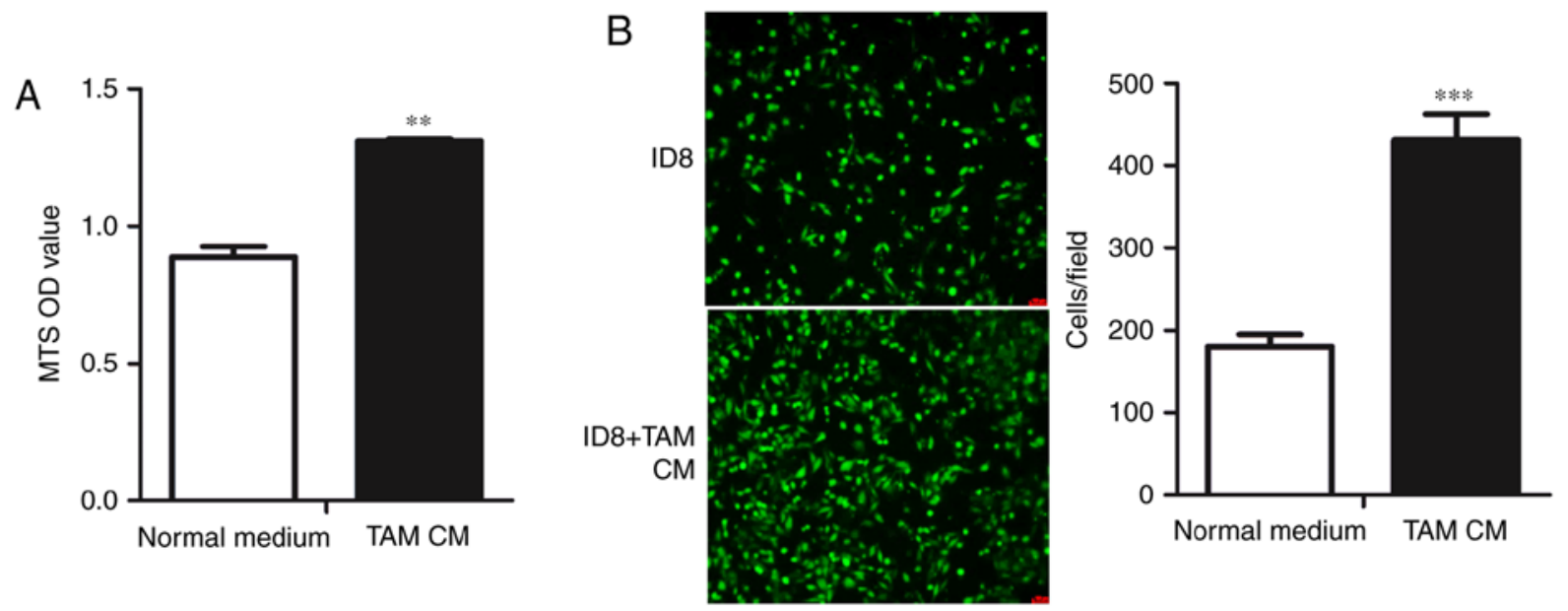

Figure 2. TAM CM promotes the proliferation and migration of EOC cells. (A) ID8 cells were cultured with normal medium or TAM CM for $72 \mathrm{~h}$. A cell proliferation assay (MTS assay) was performed to observe the effect of TAMs on the proliferation of ID8 cells. (B) After growing in normal medium or TAM CM for $48 \mathrm{~h}$, a cell migration assay was performed to assess the effect of TAMs on the migration of ID8 cells. The cells were counted $16 \mathrm{~h}$ after plating in Transwell chambers. The bar graphs represents the means \pm SDs of three independent experiments; ${ }^{* *} \mathrm{P}<0.01,{ }^{* * *} \mathrm{P}<0.001$. TAMs, tumor-associated macrophages. CM, conditioned medium. EOC, epithelial ovarian cancer. Scale bar, $100 \mu \mathrm{m}$.

OC specimens is correlated with poor prognosis, we assessed whether TAMs similarly affect the physiological activity of mouse EOC cells. We first studied the effect of TAMs on the proliferation of ID8 cells, observing that TAM CM promoted the proliferation of ID8 cells (Fig. 2A). We next determined whether TAMs could change the migration of ID8 cells, and the results showed that the migration of ID8 cells treated with TAM CM was significantly increased compared with the control group (Fig. 2B). Taken together, these data showed that TAMs could accelerate the proliferation and migration of ID8 cells.

TAMs express higher levels of IGF1 than MO cells. In a previous study, we performed a gene chip analysis to compare the expression profile of human TAMs and M0 cells, and observed that TAMs expressed significantly higher levels of IGF1 than M0 cells. We identified this result at both the mRNA and protein levels in mouse TAMs. Consistent with the gene chip analysis, the expression level of IGF1 was higher in mouse TAMs at both the mRNA (Fig. 3A) and protein levels (Fig. 3B).

Human EOC specimens express higher levels of IGFI. The IGF1 level is higher in prostate cancer and in gastric cancer compared with corresponding benign tumors $(24,25)$; thus, we examined whether EOC would have a similar phenomenon. First, we used western blot analysis to assess the level of IGF1 in fresh ovarian specimens, and observed that EOC specimens 

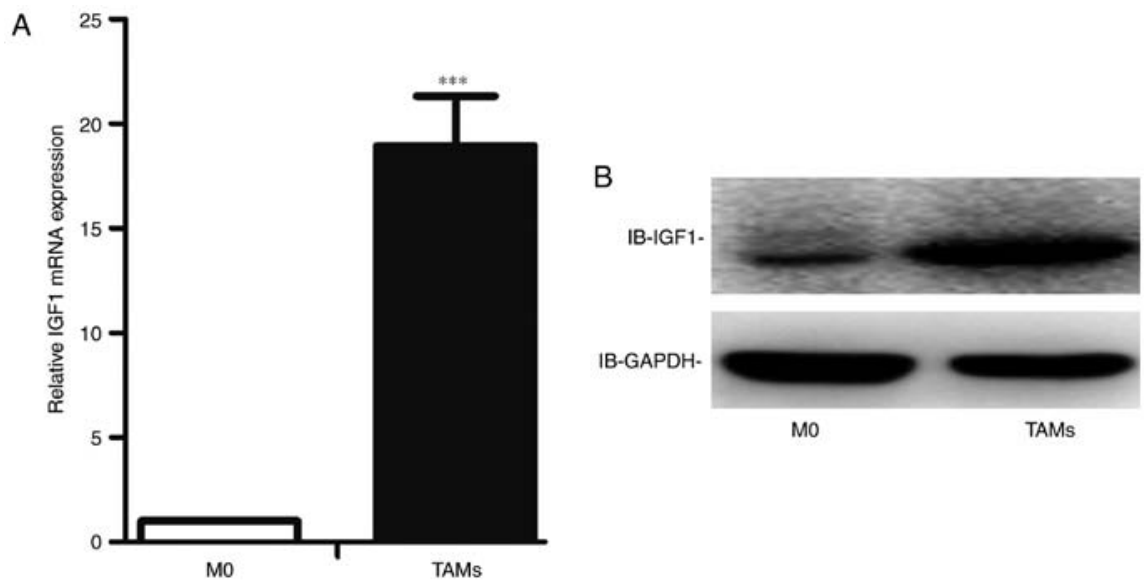

Figure 3. TAMs express higher levels of IGF1. (A and B) To verify the result of the gene chip analysis, qRT-PCR was used to compare expression levels of IGF1 between M0 cells and TAMs at the mRNA level, and western blotting was used to compare IGF1 expression at the protein level. The bar graphs represents the means \pm SDs of three independent experiments; ${ }^{* * *} \mathrm{P}<0.001$. TAMs, tumor-associated macrophages. qRT-PCR, quantitative real-time PCR. IGF1, insulin-like growth factor-1.

A
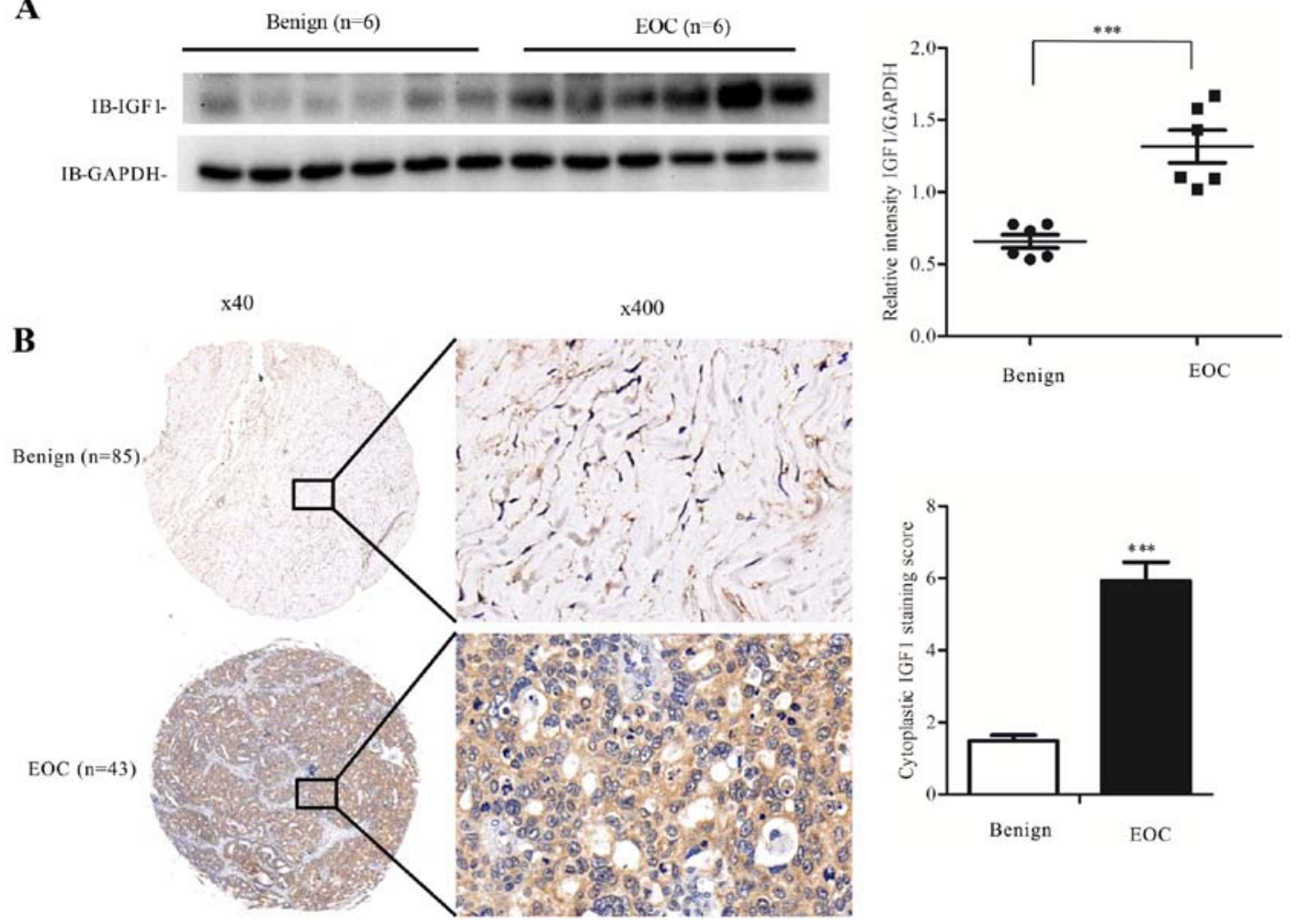

Figure 4. Human EOC specimens express higher levels of IGF1 than ovarian benign tumor tissues. (A) Western blotting was performed to compare the IGF1 level in benign ovarian tumor and EOC specimens. (B) Immunohistochemistry was used to determine the expression of IGF1 in benign ovarian tumor and EOC tissue microarrays. The bar graphs represents the means \pm SDs (A) or means \pm SEMs (B) of three independent experiments; ${ }^{* * *} \mathrm{P}<0.001$. EOC, epithelial ovarian cancer. IGF1, insulin-like growth factor-1.

expressed higher levels of IGF1 compared with levels in the benign ovarian tumors (Fig. 4A).

Next, we used IHC staining to assess the expression level of IGF1 in tissue microarrays of benign ovarian tumors and EOC. The results showed that the mean IRS score of EOC specimens was significantly higher than that of the benign ovarian tumors (Fig. 4B). Then, we classified the EOC specimens into high and low expression groups, and used the Chi-square test or Fisher's exact test to identify the correlation between clinical and pathological factors and IGF1 expression levels. The median age of the patients was 56 years. Patients with a high IGF1 expression were in a more advanced stage and tended to have undergone liver metastasis more frequently (Table I). These results indicated that high IGF1 expression may enhance the progression of EOC.

TAM CM upregulates the expression of IGFI in ID8 cells. Based on the preceding results, we determined whether TAMs 

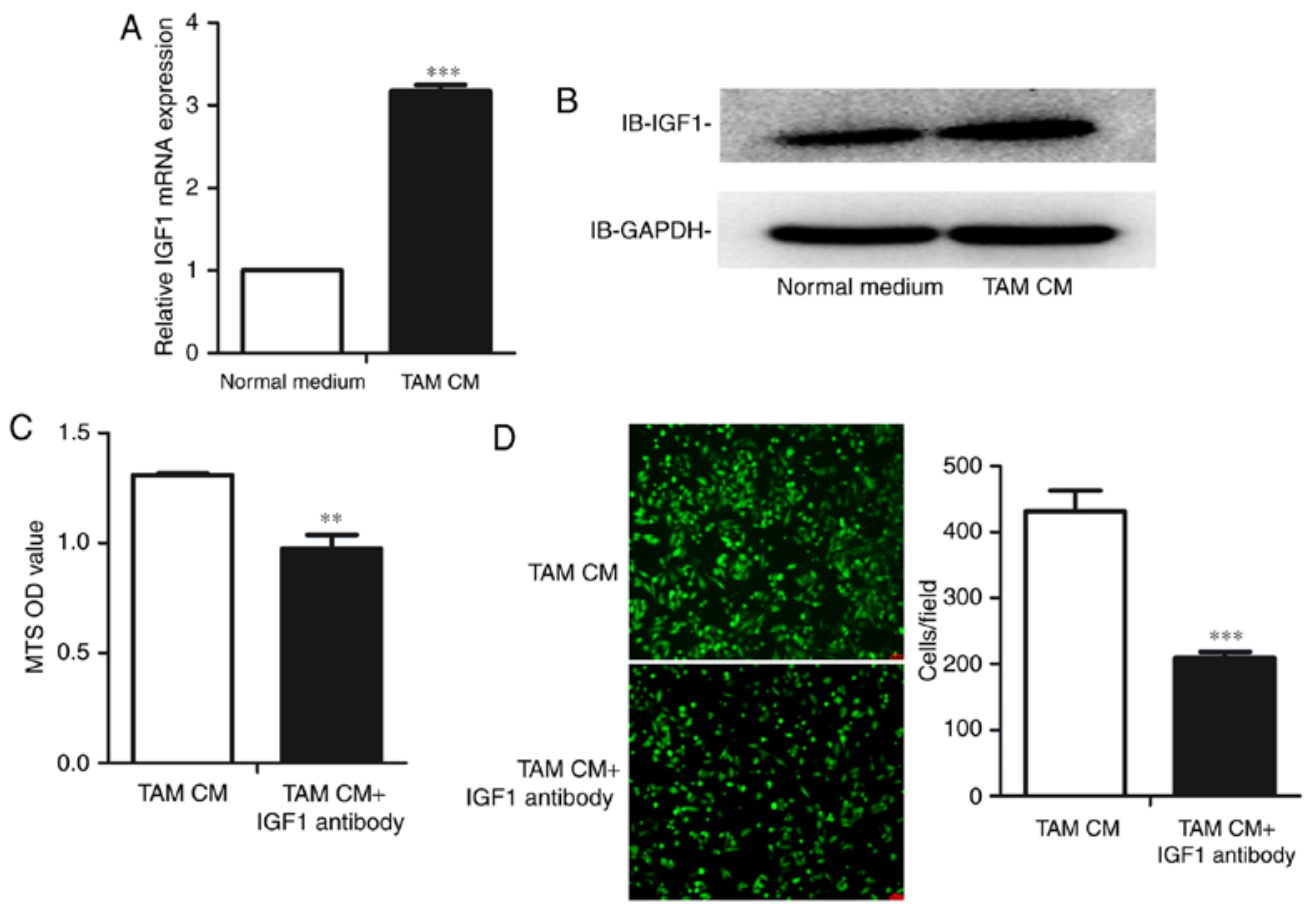

Figure 5. TAM CM upregulates IGF1 in ID8 cells, and blockade of IGF1 reverses the increase in proliferation and migration. (A and B) ID8 cells were cultured with normal medium or TAM CM for $72 \mathrm{~h}$. qRT-PCR was used to determine the effect of TAMs on expression of IGF1 at the mRNA level, whereas western blotting was used to determine IGF1 expression at the protein level. (C) ID8 cells were cultured in TAM CM or TAM CM containing IGF1 neutralizing antibody $(2 \mu \mathrm{g} / \mathrm{ml})$ for $72 \mathrm{~h}$. A cell proliferation assay (MTS assay) was used to measure the proliferation. (D) After culturing in TAM CM or TAM CM containing IGF1 neutralizing antibody for $48 \mathrm{~h}$, a cell migration assay was performed to assess the effect of TAMs on the migration of ID8 cells. The cells were counted at $16 \mathrm{~h}$ after plating in the Transwell chambers. The bar graphs represent the means \pm SDs of three independent experiments; ${ }^{* *} \mathrm{P}<0.01$,

${ }^{* * *} \mathrm{P}<0.001$. TAMs, tumor-associated macrophages. CM, conditioned medium. IGF1, insulin-like growth factor-1. Scale bar, $100 \mu \mathrm{m}$.

Table I. Correlation of IGF1 expression level with clinical and pathological factors.

\begin{tabular}{lcccc}
\hline Parameters & $\mathrm{n}$ & $\begin{array}{c}\text { Low } \\
\text { expression }\end{array}$ & $\begin{array}{c}\text { High } \\
\text { expression }\end{array}$ & P-value \\
\hline Age years) & & & & 0.749 \\
$\geq 56$ & 21 & 9 & 12 & \\
$\quad<56$ & 20 & 9 & 11 & \\
$\begin{array}{l}\text { Tumor size } \\
\geq 7\end{array}$ & 26 & 9 & 17 & \\
$<7$ & 14 & 8 & 6 & \\
$\begin{array}{l}\text { Pathologic grade } \\
\text { I+II }\end{array}$ & 12 & 6 & 6 & 0.848 \\
III & 13 & 6 & 7 & \\
Clinical stage & & & & 0.020 \\
I+II & 14 & 10 & 4 & \\
III+IV & 27 & 9 & 18 & \\
Ascites & & & & 0.951 \\
$\quad$ Yes & 23 & 10 & 13 & \\
$\quad$ No & 18 & 8 & 10 & \\
Liver metastasis & & & & 0.036 \\
$\quad$ Yes & 11 & 2 & 9 & \\
No & 29 & 16 & 13 & \\
\hline
\end{tabular}

P-value was generated by Chi-square test or Fisher's exact test, and $\mathrm{P}<0.05$ was considered as statistically significant. As clinical information was not available for each patient, the total number for each factor was different. IGF1, insulin-like growth factor-1. could alter the proliferation and migration of mouse OC cells by upregulating the tumor-promoting gene IGF1. Therefore, we treated ID8 cells with TAM CM. As expected, the expression level of IGF1 in ID8 cells increased following culture with TAM CM at both the mRNA (Fig. 5A) and protein levels (Fig. 5B).

Blockade of IGF1 reverses the alteration of proliferation and migration of ID8 cells. After observing that TAMs accelerate the proliferation and migration of ID8 cells and simultaneously upregulate IGF1, we finally assessed whether the blockade of IGF1 in TAM CM would reverse the changes in proliferation and migration. An IGF1 neutralizing antibody was added to TAM CM to block the IGF1 pathway. We observed that the blockade of IGF1 reduced the proliferation of ID8 cells (Fig. 5C). We then determined whether the IGF1 neutralizing antibody had the same effect on the migration of ID8 cells, observing that blockade of IGF1 reversed the increase in ID8 cell migration (Fig. 5D).

Inhibition of the activation of IGF1R suppresses the proliferation and migration of ID8 cells exposed to TAM CM. To validate the value of IGF1 pathway inhibition in EOC therapy, we used the IGF1R inhibitor linsitinib to inhibit the IGF1 pathway. As expected, the phosphorylation of IGF1R, Akt and Erk was significantly inhibited (Fig. 6A). Additionally, the proliferation and migration of ID8 cells were both significantly suppressed by linsitinib (Fig. 6B and C).

Taken together, these data suggest that IGF1 may be a key regulator by which TAMs promote the proliferation and 
A

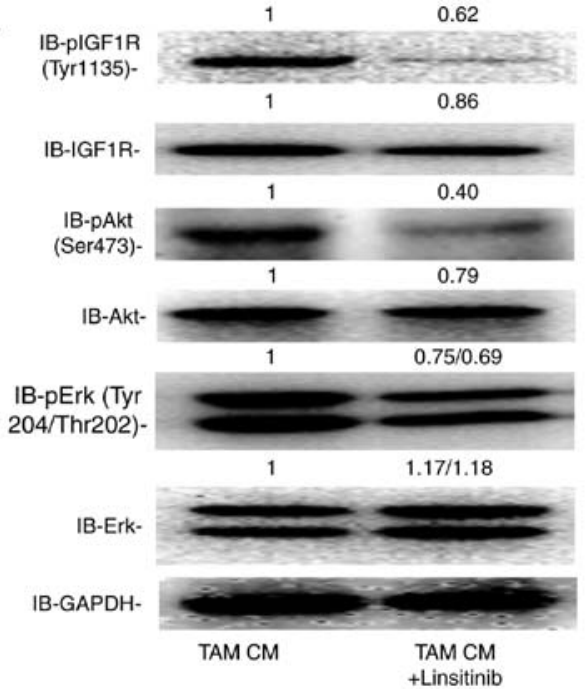

C

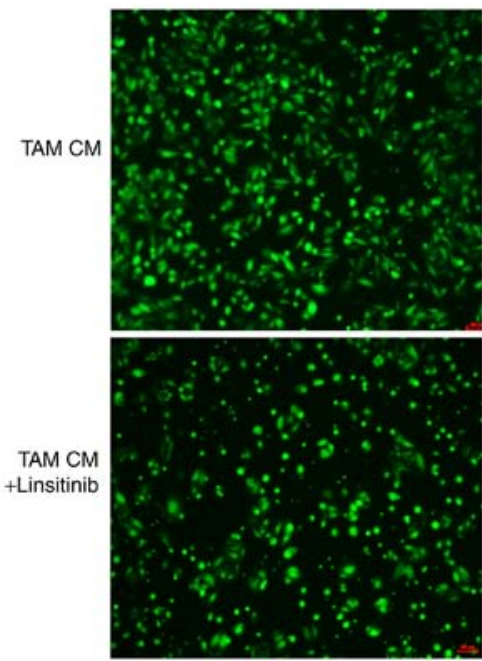

B
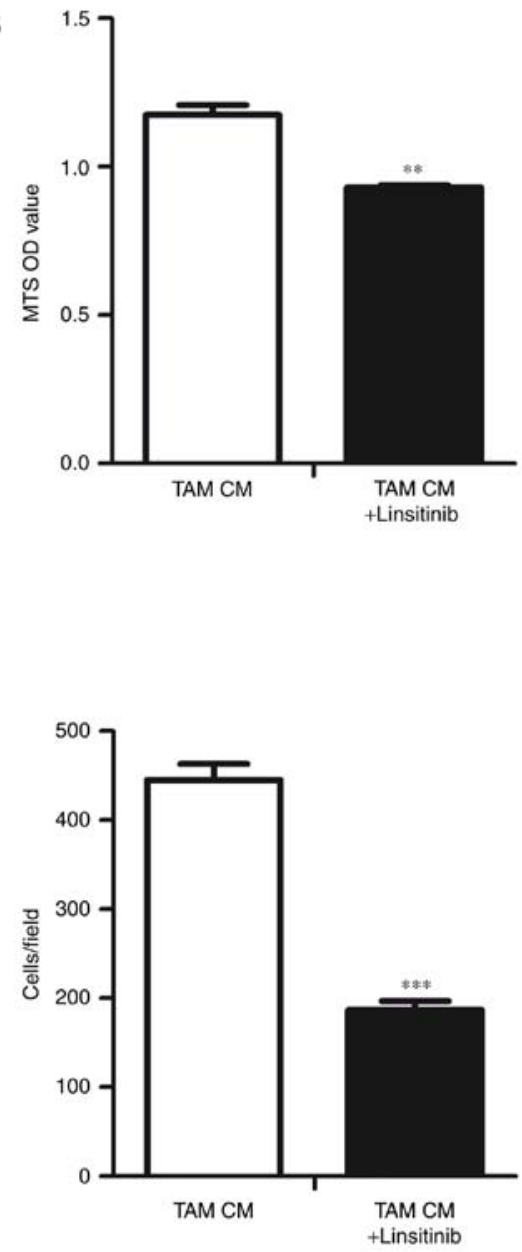

Figure 6. Inhibition of IGF1R suppresses the proliferation and migration of ID8 cells exposed to TAM CM by decreasing phosphorylated IGF1R. (A) ID8 cells were cultured in TAM CM for $48 \mathrm{~h}$, and linsitinib $(5 \mu \mathrm{M})$ was added to the medium for $24 \mathrm{~h}$. Western blotting was used to determine the protein expression of total and phosphorylated IGF1R and Akt and Erk. (B) ID8 cells were cultured in TAM CM or TAM CM containing linsitinib for $72 \mathrm{~h}(5 \mu \mathrm{M})$. A cell proliferation assay (MTS assay) was used to measure the proliferation. (C) After growing in TAM CM or TAM CM containing linsitinib for $48 \mathrm{~h}$, a cell migration assay was performed to assess the effect of IGF1R inhibition on the migration of ID8 cells. The cells were counted at $16 \mathrm{~h}$ after being plated in Transwell chambers. The bar graphs represent the means \pm SDs of three independent experiments; ${ }^{* *} \mathrm{P}<0.01,{ }^{* * * *} \mathrm{P}<0.001$. IGF1R, insulin-like growth factor- 1 receptor. TAMs, tumorassociated macrophages. CM, conditioned medium. Scale bar, $100 \mu \mathrm{m}$.

migration of OC cells, indicating the potential of IGF1 inhibition in EOC therapy.

\section{Discussion}

In the present study, we showed that IGF1 is expressed at higher levels in mouse TAMs than in M0 cells, TAMs were able to enhance the proliferation and migration of ID8 cells by upregulating IGF1, and inhibition of the IGF1R pathway effectively suppressed the proliferation and migration of ID8 cells exposed to TAM CM.

We established a mouse TAM model and observed that TAM CM accelerated the proliferation and migration of ID8 cells, supporting the role of TAMs in the progression of OC (Figs. 1 and 2). The TME is a supportive and receptive tissue microenvironment undergoing a series of molecular and cellular changes to form metastatic-designated sites, or the fertile soil in preparation for metastatic tumor cell seed colonization, thus supporting tumor settlement in distant organs and promoting tumor metastasis (6-8). As the most common immune cells in the TME, TAMs infiltrate the TME in OC in large numbers and play an important role in the formation of the OC TME and education of OC cells to develop to become more malignant by secreting various factors, such as IL-6 and IDO $(16,18,19,39,40)$. Indeed, therapeutic methods targeting TAMs have been demonstrated as effective for controlling OC. An inhibitor of IDO, which is associated with the formation of TAMs, can control the growth of OC in vivo (40). An MCSF inhibitor was found to reduce the infiltration of TAMs, and promote the survival of peritoneal blood vessels, thereby reducing the production of ascites in mice transplanted with OC cells (41).

IGF1 is a cancer progression-related gene that is associated with the growth, metastasis and chemoresistance of various cancers (23-27). In the present study, we confirmed that TAMs significantly expressed higher IGF1 levels than M0 cells as 
demonstrated in a previous gene chip analysis (Fig. 3). More importantly, the level of IGF1 was high in EOC specimens and was related to more advanced clinical stage and liver metastasis (Fig. 4; Table I). Thus, we demonstrated that TAMs promoted the progression of EOC by upregulating IGF1. As expected, these results showed that TAM CM upregulated IGF1, whereas an IGF1 neutralizing antibody reversed the alteration of proliferation and migration (Fig. 5). Additionally, these results provide evidence that IGF1 may be a key factor in the promotion of EOC progression through TAMs, explaining its high expression in EOC. Finally, we propose that the mechanism of the upregulation of IGF1 in ID8 cells through TAMs may involve exosomes, which could transfer microRNAs, mRNAs, DNA fragments and proteins, particularly miRNA, from donor cells to recipient cells (42) or secrete cytokines, which also play an important role in the communication between different cells (43). However, these hypotheses need further experimental validation.

Targeting the IGF1 pathway has been demonstrated as effective in the therapy of various cancers $(28,44)$. A typical example is that IGF1R inhibitors decreased the viability of chronic lymphocytic leukemia cells in a microenvironment context (28). Theranostic nanoparticles IGF1-IONP-Dox were found to significantly inhibit the growth of pancreatic tumors (44). Indeed, knockdown of IGF-1 using siRNA decreased the migration and invasion of ES-2 cells (45). In the present study, we used the IGF1R inhibitor linsitinib to block the phosphorylation activation of the IGF1/IGF1R/Akt and IGF1/ IGF1R/Erk pathways. Remarkably, IGF1 pathway inhibition reduced the proliferation and migration of ID8 cells, even those exposed to TAM CM, suggesting that the IGF1 pathway is potentially involved in EOC therapy, even in the TME (Fig. 6). However, whether IGF1 is effective in vivo and whether it will be more effective combined with other targets requires additional studies.

In conclusion, these results showed that TAMs play an active role in promoting the proliferation and migration of EOC, and in upregulating the expression of IGF1, whereas the blockade of IGF1 reversed the changes in proliferation and migration. These findings may represent a new mechanism related to the growth and metastasis of EOC, and currently available IGF1 inhibitors may be potential tools for EOC intervention.

\section{Acknowledgements}

The authors thank Wang Kai from the Central Lab of Shanghai First Maternity and Infant Hospital for excellent technical assistance. This study was supported by grants from the National Natural Science Foundation of China (81072136, 81372787), the Shanghai Municipal Bureau of Health (20134033), and the Shanghai Health system joint research project (2013ZYJB0201).

\section{References}

1. Torre LA, Bray F, Siegel RL, Ferlay J, Lortet-Tieulent J and Jemal A: Global cancer statistics, 2012. CA Cancer J Clin 65: 87-108, 2015.

2. Siegel RL, Miller KD and Jemal A: Cancer Statistics, 2017. CA Cancer J Clin 67: 7-30, 2017.
3. Colombo PE, Fabbro M, Theillet C, Bibeau F, Rouanet P and Ray-Coquard I: Sensitivity and resistance to treatment in the primary management of epithelial ovarian cancer. Crit Rev Oncol Hematol 89: 207-216, 2014.

4. Nagaraj AB, Joseph P and DiFeo A: miRNAs as prognostic and therapeutic tools in epithelial ovarian cancer. Biomarkers Med 9: 241-257, 2015.

5. Naora $\mathrm{H}$ and Montell DJ: Ovarian cancer metastasis: Integrating insights from disparate model organisms. Nat Rev Cancer 5: 355-366, 2005.

6. Son B, Lee S, Youn HS, Kim EG, Kim W and Youn BH: The role of tumor microenvironment in therapeutic resistance. Oncotarget 8: 3933-3945, 2017.

7. Liu Y and Cao X: Characteristics and Significance of the Pre-metastatic Niche. Cancer Cell 30: 668-681, 2016.

8. Wu JS, Sheng SR, Liang XH and Tang YL: The role of tumor microenvironment in collective tumor cell invasion. Future Oncol 13: 991-1002, 2017.

9. Richards KE, Zeleniak AE, Fishel ML, Wu J, Littlepage LE and Hill R: Cancer-associated fibroblast exosomes regulate survival and proliferation of pancreatic cancer cells. Oncogene 36: 1770-1778, 2017.

10. Sugiyama K, Kajiyama H, Shibata K, Yuan H, Kikkawa F and Senga T: Expression of the miR200 family of microRNAs in mesothelial cells suppresses the dissemination of ovarian cancer cells. Mol Cancer Ther 13: 2081-2091, 2014.

11. Kim J and Bae JS: Tumor-associated macrophages and neutrophils in tumor microenvironment. Mediators Inflamm 2016: 6058147, 2016.

12. Sica A, Larghi P, Mancino A, Rubino L, Porta C, Totaro MG, Rimoldi M, Biswas SK, Allavena P and Mantovani A: Macrophage polarization in tumour progression. Semin Cancer Biol 18: 349-355, 2008.

13. Zhang L, Zhang S, Yao J, Lowery FJ, Zhang Q, Huang WC, Li P, Li M, Wang X, Zhang C, et al: Microenvironment-induced PTEN loss by exosomal microRNA primes brain metastasis outgrowth. Nature 527: 100-104, 2015.

14. Kurahara H, Takao S, Maemura K, Mataki Y, Kuwahata T, Maeda K, Sakoda M, Iino S, Ishigami S, Ueno S, et al: M2-polarized tumor-associated macrophage infiltration of regional lymph nodes is associated with nodal lymphangiogenesis and occult nodal involvement in $\mathrm{pN} 0$ pancreatic cancer. Pancreas 42: 155-159, 2013.

15. Reinartz S, Schumann T, Finkernagel F, Wortmann A, Jansen JM, Meissner W, Krause M, Schwörer AM, Wagner U, Müller-Brüsselbach S, et al: Mixed-polarization phenotype of ascites-associated macrophages in human ovarian carcinoma: Correlation of CD163 expression, cytokine levels and early relapse. Int J Cancer 134: 32-42, 2014.

16. Lan C, Huang X, Lin S, Huang H, Cai Q, Wan T, Lu J and Liu J: Expression of M2-polarized macrophages is associated with poor prognosis for advanced epithelial ovarian cancer. Technol Cancer Res Treat 12: 259-267, 2013.

17. Pollard JW: Tumour-educated macrophages promote tumour progression and metastasis. Nat Rev Cancer 4: 71-78, 2004.

18. Takaishi K, Komohara Y, Tashiro H, Ohtake H, Nakagawa T, Katabuchi $\mathrm{H}$ and Takeya M: Involvement of M2-polarized macrophages in the ascites from advanced epithelial ovarian carcinoma in tumor progression via Stat 3 activation. Cancer Sci 101: 2128-2136, 2010.

19. Carroll MJ, Kapur A, Felder M, Patankar MS and Kreeger PK: M2 macrophages induce ovarian cancer cell proliferation via a heparin binding epidermal growth factor/matrix metalloproteinase 9 intercellular feedback loop. Oncotarget 7: 86608-86620, 2016.

20. Iams WT and Lovly CM: Molecular pathways: Clinical applications and future direction of insulin-like growth factor-1 receptor pathway blockade. Clin Cancer Res 21: 4270-4277, 2015.

21. Dyer AH, Vahdatpour C, Sanfeliu A and Tropea D: The role of insulin-like growth factor 1 (IGF-1) in brain development, maturation and neuroplasticity. Neuroscience 325: 89-99, 2016.

22. Baserga R: The contradictions of the insulin-like growth factor 1 receptor. Oncogene 19: 5574-5581, 2000.

23. Zhang M, Hu Z, Huang J, Shu Y, Dai J, Jin G, Tang R, Dong J, Chen Y, Xu L, et al: A 3'-untranslated region polymorphism in IGF1 predicts survival of non-small cell lung cancer in a Chinese population. Clin Cancer Res 16: 1236-1244, 2010.

24. Soulitzis N, Karyotis I, Delakas D and Spandidos DA: Expression analysis of peptide growth factors VEGF, FGF2, TGFB1, EGF and IGF1 in prostate cancer and benign prostatic hyperplasia. Int J Oncol 29: 305-314, 2006. 
25. Xu L, Zhou R, Yuan L, Wang S, Li X, Ma H, Zhou M, Pan C, Zhang J, Huang N, et al: IGF1/IGF1R/STAT3 signaling-inducible IFITM2 promotes gastric cancer growth and metastasis. Cancer Lett 393: 76-85, 2017.

26. Pacher M, Seewald MJ, Mikula M, Oehler S, Mogg M, Vinatzer U, Eger A, Schweifer N, Varecka R, Sommergruber W, et al: Impact of constitutive IGF1/IGF2 stimulation on the transcriptional program of human breast cancer cells. Carcinogenesis 28: 49-59, 2007.

27. Hirakawa T, Yashiro M, Doi Y, Kinoshita H, Morisaki T, Fukuoka T, Hasegawa T, Kimura K, Amano R and Hirakawa K: Pancreatic fibroblasts stimulate the motility of pancreatic cancer cells through IGF1/IGF1R sgnaling under hypoxia. PLoS One 11: e0159912, 2016

28. Yaktapour N, Übelhart R, Schüler J, Aumann K, Dierks C, Burger M, Pfeifer D, Jumaa H, Veelken H, Brummer T, et al: Insulin-like growth factor-1 receptor (IGF1R) as a novel target in chronic lymphocytic leukemia. Blood 122: 1621-1633, 2013.

29. Terry KL, Tworoger SS, Gates MA, Cramer DW and Hankinson SE: Common genetic variation in IGF1, IGFBP1 and IGFBP3 and ovarian cancer risk. Carcinogenesis 30: 2042-2046, 2009.

30. Lau MT and Leung PC: The PI3K/Akt/mTOR signaling pathway mediates insulin-like growth factor 1-induced E-cadherin downregulation and cell proliferation in ovarian cancer cells. Cancer Lett 326: 191-198, 2012.

31. Poljicanin A, Filipovic N, Vukusic Pusic T, Soljic V, Caric A, Saraga-Babic M and Vukojevic K: Expression pattern of RAGE and IGF-1 in the human fetal ovary and ovarian serous carcinoma. Acta Histochem 117: 468-476, 2015.

32. Torng PL, Lee YC, Huang CY, Ye JH, Lin YS, Chu YW, Huang SC, Cohen P, Wu CW and Lin CT: Insulin-like growth factor binding protein-3 (IGFBP-3) acts as an invasion-metastasis suppressor in ovarian endometrioid carcinoma. Oncogene 27: 2137-2147, 2008

33. Koti M, Gooding RJ, Nuin P, Haslehurst A, Crane C, Weberpals J, Childs T, Bryson P, Dharsee M, Evans K, et al: Identification of the IGF1/PI3K/NF $\kappa \mathrm{B} / \mathrm{ERK}$ gene signalling networks associated with chemotherapy resistance and treatment response in high-grade serous epithelial ovarian cancer. BMC Cancer 13 $549,2013$.

34. Huang YF, Cheng WF, Wu YP, Cheng YM, Hsu KF and Chou CY: Circulating IGF system and treatment outcome in epithelial ovarian cancer. Endocr Relat Cancer 21: 217-229, 2014.
35. Li Y, Li Y, Zhang J, Zheng C, Zhu H, Yu H and Fan L: Circulating insulin-like growth factor-1 level and ovarian cancer risk. Cell Physiol Biochem 38: 589-597, 2016.

36. Gianuzzi X, Palma-Ardiles G, Hernandez-Fernandez W, Pasupuleti V,Hernandez AV and Perez-Lopez FR: Insulin growth factor (IGF) 1, IGF-binding proteins and ovarian cancer risk: A systematic review and meta-analysis. Maturitas 94: 22-29, 2016.

37. Lin Y, Wei C, Liu Y, Qiu Y, Liu C and Guo F: Selective ablation of tumor-associated macrophages suppresses metastasis and angiogenesis. Cancer Sci 104: 1217-1225, 2013.

38. Remmele W and Stegner HE: Immunhistochemischer Nachweis von Oestrogenrezeptoren (ER-ICA) in Mammakarzinomen. Frauenarzt 28: 41-43, 1987 (In German).

39. Coward J, Kulbe H, Chakravarty P, Leader D, Vassileva V, Leinster DA, Thompson R, Schioppa T, Nemeth J, Vermeulen J, et al: Interleukin-6 as a therapeutic target in human ovarian cancer. Clin Cancer Res 17: 6083-6096, 2011.

40. Koblish HK, Hansbury MJ, Bowman KJ, Yang G, Neilan CL, Haley PJ, Burn TC, Waeltz P, Sparks RB, Yue EW, et al: Hydroxyamidine inhibitors of indoleamine-2,3-dioxygenase potently suppress systemic tryptophan catabolism and the growth of IDO-expressing tumors. Mol Cancer Ther 9: 489-498, 2010.

41. Moughon DL, He H, Schokrpur S, Jiang ZK, Yaqoob M, David J, Lin C, Iruela-Arispe ML, Dorigo O and Wu L: Macrophage blockade using CSF1R inhibitors reverses the vascular leakage underlying malignant ascites in late-stage epithelial ovarian cancer. Cancer Res 75: 4742-4752, 2015.

42. Wang Z, Chen JQ, Liu JL and Tian L: Exosomes in tumor microenvironment: Novel transporters and biomarkers. J Transl Med 14: 297,2016

43. Goyne HE, Stone PJ, Burnett AF and Cannon MJ: Ovarian tumor ascites $\mathrm{CD} 14^{+}$cells suppress dendritic cell-activated CD4 ${ }^{+} \mathrm{T}$-cell responses through IL-10 secretion and indoleamine 2,3-dioxygenase. J Immunother 37: 163-169, 2014.

44. Zhou H, Qian W, Uckun FM, Wang L, Wang YA, Chen H, Kooby D, Yu Q, Lipowska M, Staley CA, et al: IGF1 Receptor targeted theranostic nanoparticles for targeted and image-guided therapy of pancreatic cancer. ACS Nano 9: 7976-7991, 2015.

45. Ukaji T, Lin Y, Banno K, Okada S and Umezawa K: Inhibition of IGF-1-mediated cellular migration and invasion by migracin A in ovarian clear cell carcinoma cells. PLoS One 10: e0137663, 2015. 